\title{
LOS INSTRUMENTOS Y SU CONTEXTO. EL CASO DE LA MARINA ESPAÑOLA EN EL SIGLO XVIII
}

\author{
MANuEL SElLÉs \\ Facultad de Filosofia. UNED.
}

ABSTRACT: The paper deals with problems and needs derived from the acquisition and use of instrumens by an institution such as the Spanish Navy in the context of $18^{\text {th }}$ Century. In order to analize the results of the Navy instrumentation policy, particular elements are examined, such as the composition of the institution, its priorities, and different issues concerning technical and educational factors.

La historia de la instrumentación puede abarcar toda una variedad de aproximaciones, desde la que se podría denominar una historia general, que entrañaría el contexto teórico, diseño y evolución de un determinado tipo de instrumentos, hasta una historia particular, que estudiaría las vicisitudes de una pieza concreta. Entre ambos tipos de historia se insertaría la relativa a las circunstancias de la apropiación y empleo por un colectivo de un conjunto de instrumentos. Desde esta última perspectiva, en las páginas que siguen me ocupo del caso de los instrumentos implicados en la práctica y desarrollo de la navegación en el siglo XVIII, más particularmente en su segunda mitad, dentro de la coyuntura de la Marina española de la época.

La Marina española se configuró como cuerpo dotado de una oficialidad permanente a principios del siglo XVIII. Para el adiestramiento de esta oficialidad se estableció en Cádiz en 1717 la Companía y Academia de Guardias Marinas. Los mandos de la Compañía estaban constituidos por un capitán comandante, un teniente y un alférez; el profesorado de la Academia, por un Director y primer profesor de matemáticas, junto con otros seis profesores que impartían, 
respectivamente, las materias de artillería, armas, danza, construcción naval, maniobra, y uso de instrumentos náuticos. Los cadetes, con una edad entre los catorce y dieciséis años, llegaban a la Academia sabiendo leer y escribir, y se les impartía un núcleo fundamental de conocimientos matemáticos (aritmética, geometría y trigonometría), a partir del cual los más adelantados, y siguiendo sus inclinaciones, podían aprender materias tales como cosmografía, náutica, fortificación y teoría de la artillería, cuya enseñanza estaba al cargo del Director. Se trataba, así, de una formación poco exigente, lo cual se puede justificar si se tiene en cuenta que los oficiales no tenían directamente a su cargo el gobierno técnico del buque. Siguiendo la tradición, eran los pilotos quienes controlaban la ruta y marcaban regularmente la posición en la carta (una situación, no obstante, que se habría invertido hacia finales del siglo). ${ }^{1}$

Según el sistema de instrucción creado para los pilotos a principios del siglo XVI, los catedráticos de la Casa de Contratación de Sevilla, puestos bajo la dirección del Piloto Mayor, impartían clases de navegación y construían y revisaban los instrumentos; y los aspirantes a pilotos debían ser examinados y aprobados por el Piloto Mayor antes de poder ejercer su oficio. Este fue el único centro de formación náutica en Espana hasta la última parte del siglo XVII, momento en que se estableció en Sevilla el Colegio de San Telmo - concretamente, en 1681 - en donde se acogía a jóvenes huérfanos para formarlos en la marinería y en el pilotaje. Al establecerse la Academia de Guardias Marinas, el Piloto Mayor, único cargo por entonces ocupado en la Casa de Contratación, pasó a dirigir aquélla, así como también lo hizo en 1722 Pedro Manuel Cedillo, profesor del Colegio de San Telmo, quien le sucedería al año siguiente en la dirección y ocuparía el cargo hasta mediados de siglo. Con ello la Academia incorporaba a los únicos profesores de navegación que existían en ese momento en España.

Por entonces el pilotaje había experimentado algunos perfeccionamientos respecto del que se conoce como "arte de navegar" ibérico del siglo XVI. Los cálcu-

${ }^{1}$ Acerca de las vicisitudes de la ensefianza de los pilotos en la época y su pulso con la oficialidad de los buques véase M. A. SELLEs y A, LAFUENTE, "La formación de los pilotos en la España del siglo XVIII", en J. L. PESET et al (eds.), La ciencia moderna y el Nuevo Mundo, Madrid: CSIC y SEHCT, 1985, págs. 149-191. Los estudios de los guardias marinas se analizan en A. LAFUenTE y M. SEllés, El Observatorio de Cádiz (1753-1831), Madrid: Ministerio de Defensa, Instituto de Historia y Cultura Naval, 1988. Sobre el detalle de sus contenidos, M. A. SELLES, Navegación astronómica en la España del siglo XVIII, Madrid: UNED, 2000. 
los necesarios para fijar la posición geográfica del buque habían cobrado mayor rigor, introduciéndose el empleo de tablas de logaritmos y de funciones trigonométricas. En lo relativo a instrumentos, la aguja náutica (hoy, vulgarmente, brújula) se había dotado de elementos para marcar la salida y puesta del Sol con el fin de corregir periódicamente la declinación magnética; junto al cuadrante de un arco, el astrolabio náutico y la ballestilla se había introducido un nuevo instrumento para medir alturas del Sol, el cuadrante de Davis, también llamado cuadrante de dos arcos o cuadrante inglés; $y$ asimismo se usaba la corredera como elemento auxiliar para la determinación de la distancia recorrida. Por otra parte, habían aparecido las llamadas cartas esféricas, en las que se empleaba la proyección de Mercator, que comportaban un nuevo conjunto de operaciones para su manejo, pues si en ellas los rumbos constantes se representaban por líneas rectas, quedaban alteradas las distancias. $^{2}$

Era responsabilidad de cada piloto procurarse sus propios instrumentos, bien construyéndolos por sí mismo, bien adquiriéndolos a artesanos que los fabricasen. Los manuales del siglo XVI recogían sistemáticamente las instrucciones necesarias para llevar a cabo su construcción. Los de la primera mitad del siglo XVIII solían omitir ya este punto, aunque no descuidaban las destinadas a su verificación. La ausencia de información respecto a las condiciones de su adquisición hace suponer que los pilotos y oficiales no tenían especiales dificultades a la hora de surtirse de instrumentos en el país, los cuales podían fabricar artesanos de cierta habilidad. Pero en 1731 apareció un nuevo instrumento para la medición de alturas, el cuadrante de Hadley, precedente directo del actual sextante. En este caso su construcción era más delicada, y sus posteriores perfeccionamientos excluyeron por completo la posibilidad de que fuesen fabricados fuera de un grupo de constructores especializados radicados fundamentalmente en Inglaterra y, en menor medida, en Francia. Pero antes de volver sobre esto conviene examinar otras circunstancias que enmarcaron toda la andadura científica de la Marina.

Entre las características más destacadas de la ciencia española del siglo XVIII se encuentra la carencia de instituciones científicas, que motivó que este tipo de

2 Sobre la evolución de los instrumentos empleados en la práctica de la navegación, puede verse M. A. SELLEs, Los instrumentos de la navegación. Del Mediterráneo al Pactfico, Barcelona: Lunwerg, 1994. 
actividades, cuando hubo que llevarlas a cabo, quedasen en manos de las fuerzas armadas, las únicas que, a través de las Academias militares, poseían una cierta formación en estas materias, lo que se ha denominado "militarización" de la ciencia española del momento. Fue ésta una iniciativa motivada por la urgencia de los tiempos y que, al llenar en principio las necesidades básicas de la corona en materias científicas y tecnológicas, contribuyó a postergar la fundación de una Academia de Ciencias según el modelo continental. ${ }^{3}$

En el ramo de la Marina, esta situación se puso de manifiesto bastante temprano. Como es sabido, la polémica sobre la figura de la Tierra — si achatada por los polos o por el ecuador - motivó que para resolverla la Academia de Ciencias francesa enviase dos expediciones a distintas latitudes. Para llevar a cabo los trabajos de la expedición que iba a medir un arco de meridiano en las proximidades del ecuador se solicitó en 1734 permiso al gobierno español para pasar al Virreinato del Perú. El permiso fue concedido a condición de que los académicos franceses fuesen acompañados en sus trabajos por dos españoles que participasen en los mismos. Las circunstancias apuntan a que tales sujetos, «inteligentes en la matemática y astronomía», no se pudieron encontrar, lo que motivó el envío de dos guardiamarinas ascendidos para la ocasión a tenientes de navío, Jorge Juan y Antonio de Ulloa, a los que se proveyó de un conjunto de instrumentos igual al que llevaban los franceses, gestionándose su adquisición a través del académico du Fay ${ }^{4}$. Sin duda la talla científica de los dos guardiamarinas, ascendidos para la ocasión a tenientes de navío, no estaba a la altura de la de los académicos; pero constan sus buenos conocimientos matemáticos de partida y la excelente formación que adquirieron durante los años en que transcurrieron los trabajos. ${ }^{5}$

${ }^{3}$ Véase un breve panorama, con una propuesta de periodización, en A. LAFUENTE y J. L. PESET, "Las actividades e instituciones cientificas en la España ilustrada», en M. SELLES, J. L. PESET y A. LAFUENTE (comps.), Carlos III y la ciencia de la Ilustración, Madrid: Alianza, 1987, págs. 29. 79. Asimismo su "Militarización de las actividades científicas en la España ilustrada (1726-1751)", en J. L. PESET et al (eds.), La ciencia moderna y el nuevo mundo, op.cit., págs. 127-147.

${ }^{4}$ Sobre estas primera gestiones, Luis J. RAMOS, "La Expedición de la Academia de Ciencias de París para la medición del grado a la altura del Ecuador y la inclusión de 'Dos sujetos espanoles inteligentes en la matemática y la astronomía' (1734-1735)", Asclepio, XXXIX-2 (1987), 4967. La designación de un agente, bien un miembro de la Embajada o, más frecuentemente, un sujeto inteligente en la materia, constitufa entonces el procedimiento habitual para la adquisición de libros e instrumentos en el extranjero.

${ }^{5}$ Los trabajos realizados y la participación espafiola en los mismos se estudian en A. LAFUENTE y A. Delgado, La geometrización de la Tierra (1735-1744), Madrid: CSIC, 1984 y A. LAFUENTE 
$\mathrm{Al}$ retorno de Juan y Ulloa, esta formación los tornó en indispensables auxiliares para el desarrollo de los planes de renovación del país del entonces todopoderoso Marqués de la Ensenada, quien los comisionó, Juan a Inglaterra, Ulloa a Francia y a otras naciones europeas, con misiones de información en materias cientificas y tecnológicas ${ }^{6}$. A su retorno, Juan fue puesto al frente de la Compafía de Guardias Marinas, para la que por su mediación se había contratado a uno de los académicos franceses, Louis Godin, como Director de la Academia, y se reformaron en profundidad los estudios. Desde el punto de vista de la instrumentación, importa señalar la descripción, realizada por primera vez en castellano, del octante de Hadley, la contribución de Juan a su estudio teórico y, sobre todo, el establecimiento de un Observatorio astronómico anexo a la Academia. ${ }^{7}$

Los proyectos que Juan realizó por estos años supusieron un punto de inflexión en la política cientifica española y sentaron un precedente que serviría de referencia en décadas sucesivas. No todos pudieron llevarse a término. Su proyecto de reglamento para una Academia de Ciencias quedó sin efecto al no establecerse ésta, así como también su plan de trabajos para la triangulación geodésica del territorio con vistas al trazado de una carta de España. Mejor fin tuvieron su programa de construcción naval, que comenzó a aplicarse en los arsenales de los recientemente creados Departamentos marítimos, y la fundación del Observatorio de Cádiz, cuyos instrumentos estaban en posición en 1753. Sin contar con que la racionalidad que introdujo en los estudios de navegación ya no se iba a abandonar.

El origen del Observatorio está en el plan del marqués de la Ensenada de enviar con Juan a Londres a dos guardiamarinas con la misión de aprender matemáticas y física. Finalizados estos estudios, uno de ellos debía permanecer allí

y A. MAzUecos, Los caballeros del punto fijo. Ciencia, política y aventura en la expedición geodésica bispanofrancesa al virreinato del Perí en el siglo XVIII, Barcelona: Serbal/CSIC, 1987.

${ }^{6} \mathrm{~A}$. LAfuente y J. L. PeseT, "Política cientifica y espionaje industrial en los viajes de Jorge Juan y Antonio de Ulloa (1748-1751)", Melanges de la Casa de Velazquez, XVII (1981), 233-262.

${ }^{7}$ La descripción se encuentra en J. JUAN y A. de UlLOA, Relación histórica del Viaje a la América Meridional hecho de orden de S. M. para medir algunos grados de Meridiano Terrestre, y venir por ellos en conocimiento de la verdadera Figura y Magnitud de la Tierra, con otras varias Observaciones Astronómicas, Madrid, 1748, Lib. IV, Cap. I, "Appendiz a este capitulo. En que se dà noticia de un nuevo instrumento para tomar Alturas en la Mar, y de sus particulares ventajas à los que antes se usaban en la Navegación.. El estudio de Juan se encuentra en su Compendio de Navegación para el uso de los Cavalleros Guardias-Marinas, Cádiz, 1757. 
como tutor de un nuevo guardiamarina, estableciéndose así una cadena de pensionados que realizasen estos estudios. Como Juan le señaló, no había entonces en Londres - ni en ninguna otra parte- instituciones o individuos que impartiesen el tipo de formación avanzada que se deseaba. En su lugar propuso desviar esta inversión hacia la adquisición de instrumentos para establecer un Observatorio en Cádiz, en el cual podría llevarse a cabo esta enseñanza bajo la dirección de Louis Godin. Se resolvía así la dependencia del extranjero respecto de la consecución de estudios avanzados por una parte del colectivo de marinos, cuya conveniencia había puesto de manifiesto la formación adquirida por Juan y Ulloa. Otra cosa, como se verá, era lograr la independencia en el ramo de la instrumentación.

En ese momento, la dotación de instrumentos de la Academia de Guardias Marinas, mantenida con propósitos docentes, era más que modesta: en el ramo de la astronomía y la náutica, contaba con una esfera armilar, 2 globos, celeste y terráqueo, "viejos", un anillo astronómico, un astrolabio de bronce, tres cuadrantes, una ballestilla, dos láminas de latón con rosas náuticas y tres agujas de marcar. Respecto a las adquisiciones de Juan en Londres, no se ha hallado la lista concreta, pero debió desviarse poco del conjunto de instrumentos descritos algunos años después. La pieza principal era un cuarto de círculo mural de 6 pies de radio construido por John Bird. Junto a él, en la relación figuraban, entre otros de menor importancia, un cuarto de círculo móvil de 2 pies del mismo constructor, un péndulo de John Ellicott, un anteojo acromático de John Dollond con 4 pies de foco y montura ecuatorial (máquina paraláctica, según la nomenclatura de la época), dotado además de un retículo de Bradley y un heliómetro acromático del mismo Dollond, dos telescopios reflectores, uno de Short de 48 pulgadas de foco y otro de Nairne, de 40; también un anteojo de pasos, posiblemente construido por Bird, y un cuarto de círculo móvil de George Adams. Un inventario posterior añade a esta dotación otros dos telescopios de Short de 24 y 18 pulgadas de foco, respectivamente. Para la época, pues, el Observatorio de Cádiz disponía de una magnífica dotación instrumental. ${ }^{8}$

Existe constancia de que en un primer momento se realizaron trabajos relacionados con el calado (puesta en posición) del cuadrante mural y del instru-

${ }^{8}$ Las visisitudes del Observatorio hasta el primer tercio del siglo XIX se analizan en $\mathrm{A}$. LAFUente y M. Sellés, El Observatorio de Cádiz...,op. cit. 
mento de pasos, de modo que el Observatorio debió quedar pronto dispuesto para las observaciones. Sin embargo, tras este período inicial de actividad, éstas quedaron prácticamente en suspenso hasta dos décadas después. Los motivos fueron varios. Uno, los intereses particulares de L. Godin, embarcado en trabajos más relacionados con la recopilación de observaciones que con la realización de otras nuevas. Otro, las continuas comisiones de Juan, quien estuvo muy ocupado con diversos encargos y particularmente con la puesta en marcha de los Arsenales y del nuevo programa de construcción naval. Y también, pero no en último lugar, la ausencia de vinculación del Observatorio con un programa de trabajo concreto que estuviese conectado con las necesidades del momento.

En realidad, por entonces las circunstancias para el desarrollo de actividades en el Observatorio no presentaban el cariz más favorable. El marqués de la Ensenada cayó en desgracia en 1754, y los años de paz del reinado de Fernando VI (1746-1759) finalizaron pronto, cuando reinando ya Carlos III se firmaba con Francia el Tercer Pacto de Familia en 1761 y se reanudaban las hostilidades con Inglaterra y Portugal al año siguiente. La guerra terminó, con un saldo negativo para España, en 1763. Más tarde, con ocasión de la independencia de las colonias inglesas en América, se iniciaría otra guerra que se extendería desde 1779 a 1783; sin contar con la campaña de Argel en 1774. Durante estos años fue necesario aumentar el número de buques y, consiguientemente, de oficiales, creándose para ello dos nuevas Compañías de Guardias Marinas en Ferrol y Cartagena en 1776. De hecho, al margen de su intervención en enfrentamientos bélicos, la Marina tenía que reforzar su control sobre las rutas americanas, abiertas ahora por el Reglamento de Libre Comercio a los diferentes puertos peninsulares, en un proceso que, iniciado en 1765 , se remataba en 1778 y acababa con el viejo sistema de flotas. Durante esos años, pues, el objetivo principal de estas Academias fue la docencia. Por otra parte, al trasladarse en 1769la Compañía y Academia de Cádiz a la vecina Isla de León, el Observatorio quedó aislado en su antigua localización. Aun en tales circunstancias Vicente Tofiño, cuando llegó al puesto de Director de la Academia, acompañado de otro de sus profesores, José Varela, llevó a cabo un programa más o menos sistemático de observaciones entre 1773 y 1776; pero debieron cancelarlo al reclamárseles una mayor dedicación a la enseñanza.

Cuando ocupó la comandancia de la Compañía de Guardias Marinas, Juan había dirigido sus miras a la formación de algunos "oficiales científicos" que, con una instrucción superior, pudiesen sentar plaza de maestros en la Academia o 
desempeñar comisiones de carácter científico. Éstas no faltaron, debiendo ejecutarse tanto por cuestiones de prestigio nacional como por asuntos de relevancia política. Así, cuando desde la Academia de Ciencias francesa se solicitó permiso para que el abate Chappe d'Auteroche pasase a California para la observación del tránsito de Venus por el disco solar en 1769, fueron comisionados los marinos Salvador Medina y Vicente Doz para acompañar a la expedición, entrenándose en el Observatorio. En esta ocasión se adquirió en Londres un anteojo acromático, proveyendo el Observatorio de los demás instrumentos?. Por otra parte, dada la índole de su formación en matemáticas y astronomía, los marinos fueron reclamados a participar en las llamadas "expediciones de límites", encargadas de fijar las fronteras entre las posesiones españolas y portuguesas en tierras americanas. Las primeras mediciones se efectuaron a consecuencia del Tratado de Madrid de 1750; posteriormente fue necesario fijar de nuevo los límites tras el Tratado de San Ildefonso de 1777. En la primera ocasión los trabajos se extendieron entre 1754 y 1760, momento en que España denunció el tratado; la segunda expedición, dirigida por José Varela, comenzó sus tareas en 1783, prolongándose éstas hasta finales del siglo. En esta ocasión se realizó otra importante adquisición de instrumentos en Londres, a través del constructor y agente de origen portugués J. H. Magellan. En 1778, primero Portugal, tres meses después España, encargaron a Magellan la adquisición, respectivamente, de cinco y seis colecciones de instrumentos adaptados al intento, hasta el punto de prevenir su almacenamiento en pares de cajas de peso ajustado para que pudiesen ser transportadas a lomos de mulos o de caballos.

Todas las colecciones contenían, aproximadamente, la misma dotación instrumental. En la colección n. ${ }^{\circ} 1$ de las seis enviadas a España se incluían dos cuartos de círculo de dos pies de radio de Sisson (con un precio, cada uno, de 136 libras inglesas, más 15 guineas en mejoras); un péndulo astronó-

${ }^{9}$ Medina y Doz llevaban un péndulo de Ellicott, un cuarto de círculo de Bird de dos pies de radio, un anteojo ordinario - no acromático- de 14 pies, con máquina paraláctica, y un micrómetro de construcción inglesa, además de un acromático de 10 pies - posiblemente el encargado a Londres - para el que se construyó en Cádiz una máquina paraláctica. "Observación del paso de Venus por el disco Solar, ejecutada por orden de S. M. en California, por los capitanes de Fragata Dn. Salvador Medina y Dn. Vicente Doz en 3 de junio del año de 1769", MN (Museo Naval, Madrid), ms. 147, ff. 32-38; los instrumentos, en fo. 34 r. Asimismo en Doyce B. NuNIS, Jr., The 1769 Transit of Venus. The Baja California observations of Jean-Baptiste Chappe dAuteroche, Vicente de Doz, and Joaquin Veldzquez Cardenas de León, Los Angeles: Natural History Museum, 1982, págs. 119 ss. 
mico, con mecanismo de escape según el diseño de Graham, construido por Grignion; un anteojo acromático con un objetivo de tres piezas y tres pies y medio de foco, con oculares para uso terrestre y celeste, fabricado por Dollond, y provisto de un micrómetro filar; otro anteojo más pequeño, también de Dollond; una cadena de 100 pies de longitud; un barómetro, con tubos de vidrio de repuesto, y dos termómetros; un instrumento de reflexión; un compás o aguja náutica, con barras para proceder a su cebado; un estuche de instrumentos de matemáticas; un teodolito de Adams; un reloj de bolsillo de segundos; dos brújulas de bolsillo; y otros útiles de menor entidad. Todo ello empaquetado en diez cajas ${ }^{10}$. Algunas de estas colecciones quedaron depositadas en el Observatorio, convirtiéndolo así en un importante depósito de instrumentos.

Al margen de su dotación instrumental, estas comisiones, claro está, requerían personal especializado en tareas de índole geográfica y astronómica. También, por otro lado, la navegación conocía en esos momentos importantes novedades que no se podían pasar por alto. Por fin se había podido resolver el problema de la determinación de la longitud en el mar, y ello no sólo mediante un método, sino mediante dos, ambos propuestos mucho tiempo atrás, pero imposibles de llevar hasta entonces a la práctica por dificultades de indole cientifica y técnica. Uno de ellos era el conocido como de las «distancias lunares", y se basaba en la observación desde el buque de la distancia angular del borde de la Luna al Sol o a alguna estrella zodiacal de entre un conjunto preseleccionado. Esta distancia se comparaba con la previamente calculada para ese momento observada desde un meridiano de referencia, recogida en tablas de publicación anual, con el fin de establecer la diferencia horaria entre este meridiano y el correspondiente a la posición del buque. El otro método se basaba en el transporte de un cronómetro marino que conservase la hora del meridiano de partida, a fin de compararla asimismo con la hora local determinada desde el buque.

La historia de los primeros cronómetros marinos es bien conocida: fue el relojero inglés John Harrison quien finalmente ganaría con su cronómetro

${ }^{10}$ Los detalles e instrucciones para su montaje y uso, junto con algunos tratados, los recoge Magellan en su Collection des différens traités sur des instrumens d'astronomie, physique, etc., divisée en deux parties..., París y Londres, 1775 y 1780. 
n. ${ }^{\circ} 4$ el famoso premio de 20.000 libras ofrecido en 1714 por el Parlamento inglés a quien resolviese el problema de la longitud ${ }^{11}$. Pero también los relojeros franceses estaban haciendo progresos en este sentido, y con el fin de probar los cronómetros de Pierre le Roy y Ferdinand Berthoud se realizaron diversos viajes. Las expediciones de Cassini (1768-69), Fleurieu (1768-69) y Verdun, Borda y Pingré (1771-72) recalaron en Cádiz, y allí establecieron contacto con el profesorado de la Academia. El resultado fue un relanzamiento del Observatorio al escenario internacional, tras los años de abandono y olvido que sucedieron a la muerte de Godin, acaecida en 1760. En este punto merece la pena citar a Jean-Dominique Cassini (Cassini IV) durante su recalada en Cádiz en 1768:

J'aurois pu me dispenser de faire transporter à Cádiz mes instruments; j'en trovai dans l'observatoire de la marine un grand nombre de toute espece et de la meilleure construction (...) je trouvai, quand j'y arrivai, les instruments dérangés et un peu negligés; mais $\mathrm{M}$. Tofiño (...), se préparoit à rétablir l'ordre, et à y commencer des observations qui seront d'autant plus utiles que l'on en a peu de bonnes faites dans cette ville. ${ }^{12}$

Tofiño y Varela se vieron así estimulados a iniciar un programa de observaciones pero, como se ha mencionado, tuvieron que abandonarlo al reprochárseles el descuido de sus tareas docentes. El cultivo de la astronomía, por sí misma, no se contaba por entonces entre los objetivos prioritarios de la Marina. Pero, como se verá, no faltarían otros trabajos. Entre tanto, y desde un primer momento, el ministerio español estuvo al tanto de los progresos realizados en cronometría de longitudes. Durante su estancia en Londres, Jorge Juan informó haber visto el cronómetro de Harrison, y en 1765 redactó un largo informe sobre la conveniencia de que Espańa no desaprovechase cualquier oportunidad de obtener estas máquinas. En 1774 y 1776 se adquirieron a F. Berthoud sus cronómetros números $7,9,10$ y 12 , en una primera remesa, y en una segunda

"Los primeros pasos de la cronometría de longitudes se recoge en todas las historias de la tecnologra. Una obra clásica es la de R. T. Gould, The Marine Chronometer. Its History and Development, Londres, 1925. Reed. Woodbridge, Sufflok: Antique Collector's Club, 1989.

${ }^{12} \mathrm{~J}$. D. CASSINI, Voyage fait par ordre du roi en 1768 pour éprouver les montres marines inventés par M. Le Roy (...), París, 1770, pág. 43. Los viajes de prueba franceses se analizan en J. LE BoT, Quand l'art de naviguer devenait science. Les chronometres de marine français au XVIIT siècle, Grenoble: Terre et Mer, 1983. 
los 13, 14, 15 y 16, que fueron depositados en el Observatorio de Cádiz. También consta la adquisición, por esas fechas, de los relojes números 3,5 y 6 de John Arnold ${ }^{13}$. En noviembre de 1788 existen datos relativos a la adquisición de su n..$^{\circ} 61$, y un año después constan en el Observatorio los números 71,344 y 351 del mismo constructor. La Marina española, pues, tuvo desde el primer momento una buena dotación de cronómetros. Unidos a los mencionados anteriormente, formaron una muy importante colección de instrumentos para cuya formación se había reparado poco en gastos ${ }^{14}$. Diego Rostriaga, maquinista de los Reales Estudios de San Isidro de Madrid, evaluaba, c. 1774, en un millón de reales el coste global de la dotación instrumental de la Academia de Guardias Marinas $^{15}$. Otro testimonio lo suministraba Cosme Churruca en 1791, cuando escribía que, según le había manifestado otro oficial de la Armada (Magdonel, capitán de navío que mandó el Rayo en Trafalgar),

habiendo ido, en Londres, a casa de Nairne y Blunt (...), tratándose, después, de la ocupación que los extranjeros daban a los artífices ingleses, dijeron ellos que era la Espańa, y particularmente la Marina, quien les daba más ganancia, $y$, finalmente, que apenas había en aquella capital artífice de fama que no tuviera encargos de España. ${ }^{16}$

El depósito en el Observatorio de todos estos instrumentos, añadidos a los propios del mismo que fueran adquiridos por Juan, requería de personal especializado en su mantenimiento, capaz de efectuar cuanto menos pequeñas reparaciones. El puesto de instrumentario del Observatorio lo ocupaba Francisco Sierra, que mal que bien pudo ir atendiendo a dicho mantenimiento en una primera etapa en la que los instrumentos eran poco usados. En 1787, vacante el puesto, lo ocupó primero Juan Martínez, hijo de un operario del obrador de instrumentos del Arsenal, quien iba a ser asesorado por su padre, Francisco Martínez, que sería

${ }^{13}$ El no 5 aún se conserva en el Museo Naval de Madrid.

${ }^{14}$ Véase un extracto de los inventarios en F. J. GONZALEZ GONZALEZ, Instrumentos cientificos del Observatorio de San Fernando (siglos XVIII, XIX y XX), Madrid: Ministerio de Defensa, Instituto de Historia y Cultura naval, 1995.

${ }^{15}$ V. Gujarro Mora, Los instrumentos de la ciencia ilustrada. Fisica Experimental en los Reales Estudios de San Isidro de Madrid (1770-1835), Madrid: UNED, 2002, pág. 124.

${ }^{16}$ Citado por S. Garcfa Franco, Historia del arte y ciencia de navegar, 2 vols., Madrid, 1947, vol. 1, pág. 256. 
finalmente quien ocupase el puesto. Parece que su trabajo no resultó todo lo satisfactorio que se esperaba ${ }^{17}$. Esta carencia de buenos técnicos de mantenimiento, como se verá, se puso de manifiesto muy especialmente en el caso de los primeros cronómetros marinos.

Entre tanto, en 1783, finalizadas las hostilidades con Inglaterra, las actividades científicas de la Marina cobrarán un nuevo sesgo. Por esos años se echaban ya a faltar esos "oficiales cientificos" que había buscado formar Juan y sobre los que gravitó el desempeño de las comisiones que se habían dado en las últimas décadas. Por ello se tomó la decisión de inaugurar en las tres Academias de Guardias Marinas un curso avanzado, llamado de «Estudios Mayores», destinado a algunos oficiales de la Armada. En Cádiz, centro principal de la formación de estos oficiales, tales estudios supusieron la reactivación de las actividades en el Observatorio, ahora constituido en una escuela de astronomía donde se practicaban las observaciones según un plan sistemático. A la vez, desde el gobierno se lanzaba un vasto programa de indagación hidrográfica. En el punto CXCI de la «Instrucción reservada para la dirección de la Junta de Estadom, redactada por el conde de Floridablanca, se decía,

que así como de mi orden se ha pasado ahora a reconocer todo el estrecho de Magallanes, se hagan también progresivamente reconocimientos de todas las costas de mis vastos dominios en las cuatro partes del mundo, $y$ las posibles experiencias para descubrir los rumbos más cortos y más seguros de navegación a los países más distantes y menos frecuentados, ejecutándose, a lo menos cada año, uno de estos proyectos (....). ${ }^{18}$

A esas alturas esos trabajos se habían hecho realmente necesarios. La introducción de los nuevos métodos de determinación de la longitud vino a coincidir, más o menos, con el inicio de una etapa de exploraciones del Pacífico abierta por ingleses y franceses tras la Guerra de los Siete Años, quienes a la gloria de la ciencia no dejaban de unir la más práctica búsqueda de rutas co-

17 A. Lafuente y M. A. Selles, El Observatorio..., op. cit., págs. 269 ss. Se sabe muy poco sobre estos talleres de instrumentos emplazados en los Arsenales, aparte de que en ellos, como manifiesta el mismo Francisco martínez, se fabricaban agujas náuticas. Todo hace suponer que estos obradores no estaban capacitados para trabajos algo más especializados.

${ }^{18}$ En Andrés MURIEl, Historia de Carlos IV, Madrid, 2 vols., 1959, en la Biblioteca de Autores Espafioles. La cita, en el vol. 115, pág. 352. 
merciales y de posibles asentamientos coloniales en la zona ${ }^{19}$. Al gobierno español le interesaba, pues, reforzar con cierta urgencia su control marítimo y territorial.

A partir de ese momento, y hasta finales del siglo, la Marina sostuvo ininterrumpidamente expediciones hidrográficas. La primera fue inmediata: Vicente Tofino, junto con la primera dotación de oficiales destinados a ampliar estudios en el Observatorio, fue comisionado para cartografiar las costas peninsulares ${ }^{20}$. Acto seguido se acometía la empresa en las americanas. Desde el punto de vista de la instrumentación, esto supuso nuevas y no pocas adquisiciones. Por ejemplo, por citar un caso destacado, en la conocida expedición de Alejandro Malaspina (1789-94) se solicitó la adquisición a Londres de los siguientes instrumentos: un cuarto de círculo de Sisson de pie y medio de radio, dos megámetros de Ramsden, adaptados para uso geodésico, dos agujas de inclinación, tres teodolitos, cuatro anteojos acromáticos celestes, dos cronómetros de longitud de Arnold, con sus acompañantes, diez anteojos terrestres, otros dos para uso nocturno y otros cuatro de bolsillo, diez y seis sextantes o quintantes de los mejores constructores, otros tantos estuches de dibujo, un barómetro marino y otros dos barómetros para la determinación de altitudes según el diseño de De Luc. Petición que se verá todavía reforzada con heliómetros para dos de los anteojos, otro barómetro marino y dos acromáticos con montura ecuatorial. Además de la primera colección de Magellan. De hecho, no todos los instrumentos solicitados estuvieron dispuestos a la hora de la partida, y así la lista de los realmente empleados difiere un tanto de la anterior (por ejemplo, se emplearon los cronómetros marinos números 10 y 13 de Berthoud y los 61,71 y 72 de Arnold), pero con ello se tendrá una buena idea de la dotación de la que podía llegar a disponer una expedición de este tipo ${ }^{21}$. Los resultados de este esfuer-

19 Dejando aparte el viaje del comodoro Anson, que puede considerarse una expedición militar, antes de los conocidos viajes de Cook se contabilizan los de Byron (1764-66), Wallis y Carteret (1766-69) y Bougainville (1766.-69).

${ }^{20}$ Los resultados se publicarían en el Atlas maritimo de España, Madrid, 1789, el Derrotero de las costas de España en el Mediterráneo... (Madrid, 1787) y el Derrotero de las costas de España en el océano Atlántico..., Madrid, 1789.

${ }^{21}$ M. SELLES, "La preparación científica e instrumental de la expedición Malaspina», en A. Orozco, La expedición Malaspina (1789-1794). Bicentenario de la salida de Cadiz, Cádiz: Real Academia Hispanoamericana, 1991, págs. 69-81. 
zo hidrográfico se centralizaron en una nueva institución, el Depósito Hidrográfico, que establecido en 1789 como mero almacén, cobró actividad a partir de 1797 al ser nombrado director del mismo José de Espinosa Tello, pasando a encargarse de la publicación de cartas náuticas, derrotas, memorias y otras obras de interés para la navegación. Sin insistir en el tema, ya tan transitado, de las expediciones hidrográficas, merece la pena citar finalmente la evaluación del astrónomo J.B. J. Delambre, quien en 1810 escribía que:

C'est en Espagne, et par les soins de son Gouvernement, que la géographie a fait les plus des progrès depuis $1789 .{ }^{22}$

Con ello la utilización del Observatorio encontró finalmente acomodo dentro de los programas de indagación de la Marina, que buscaban atender a las necesidades propias de su instituto antes que a la práctica de la astronomía, aunque no por ello dejaron de efectuarse y comunicarse las observaciones señaladas y de aspirarse al establecimiento de un programa regular de tareas, algo dificultado por el tránsito de diferentes oficiales por el mismo. En todo caso, esta última etapa, a juzgar por los informes emitidos por el personal allí destinado, supondría el final del período de utilidad de la dotación inicial de instrumentos adquiridos por Juan, ya deteriorados y necesitados no tanto de reparaciones como de sustitución, algo que las dificultades de los tiempos demorarían hasta bien entrado el siglo XIX. Sí se logró, sin embargo, la construcción de un nuevo Observatorio en la Isla de León, cuya primera piedra se puso en 1793 , y que sigue en uso en la actualidad.

La última parte del siglo vio así ponerse definitivamente en marcha los proyectos ilustrados de renovación científica del país. En Madrid, Floridablanca proyectaba instituir finalmente una Academia de Ciencias, para la que se construyó el edificio que alberga actualmente al Museo del Prado, y que junto con el Observatorio astronómico, asimismo de nueva construcción, y el Jardín Botánico, debían constituir el complejo científico madrileño. Para dicho Observatorio se adquirió a W. Herschel un relescopio de 20 pies de foco, que desgraciadamente no llegó a entrar en pleno funcionamiento (fue quemado por los franceses, quienes emplearon el Observatorio como polvorín, cuando la

22 J. B. J. DELAMBRE, Rapport historique sur les progrès des sciences mathématiques depuis 1789 et sur leur état actuel, París, 1810, pág. 228. 
ocupación de Madrid). También se estableció una Escuela de Instrumentación dependiente del mismo, y se volvió a retomar el proyecto del mapa de España, creándose con tal fin un Cuerpo de Ingenieros Cosmógrafos. Por el lado tecnológico, se estableció una Escuela de Caminos y un Gabinete de Máquinas. La ciencia española comenzaba a despegarse así de la tutela de las fuerzas armadas, que la habían sostenido con no poco esfuerzo a lo largo del siglo. Pero, en el caso de la Marina, esto no implicaba renunciar a sus propios y ambiciosos proyectos, que suponían la fundación en la Isla de León de un complejo científico propio del que formaría parte el nuevo Observatorio y que comprendería un Museo desdicado a todos los temas relacionados con la navegación, para el que se comenzaron a realizar en el extranjero importantes inversiones. Los malos tiempos que sobrevinieron abortaron todos estos proyectos, ya en vías de realización. Y ciertamente el esperado Fernando VII hizo después bien poco por recuperarlos.

Si el tema de la aparición de los cronómetros marinos y la adquisición de algunos de los primeros de ellos por parte de la Marina española ha servido aquí de introducción para señalar el importante depósito de instrumentos que se efectuó en el Observatorio, convertido así junto con su dotación de oficiales en formación en centro neurálgico del programa expedicionario español, la puesta a punto del método de las distancias lunares sirve para introducir otra dimensión. Para la práctica del método se necesitaba, como se mencionó más arriba, disponer de tablas que predijesen las distancias de la Luna a un conjunto de astros seleccionado en el meridiano de referencia con algo así como un año como mínimo de antelación. Estas tablas surgieron de un lugar inesperado, de Gotinga, donde fueron elaboradas por el astrónomo Tobias Mayer apoyándose en sus observaciones y en el trabajo teórico de L. Euler sobre el movimiento lunar. A partir de estas tablas, el astrónomo real inglés Nevil Maskelyne impulsó la publicación de unas efemérides náuticas, el Nautical Almanac, cuyo primero ejemplar vio la luz en 1766. Francia, por su parte, recogió dichas tablas, trasladando de meridiano los valores alli tabulados, a partir de 1774. La Marina española, al igual que en el caso de los cronómetros, estuvo avisada tempranamente del surgimiento de este método, para el que sólo se necesitaban - aparte de los conocimientos teóricos necesarios para su aplicación- de estas tablas y de un sextante. Tanto las tablas como el instrumento se podían adquirir en el extranjero. La cuestión aquí no era tanto la incorporación del método a la Marina española - se comenzaron a dar clases específicas sobre el mismo en Cádiz en 1773, incorporándose más tarde la materia en los planes de estudio-, sino la elimi- 
nación de esta dependencia del extranjero. Convenía que las efemérides no sólo se refiriesen al meridiano del Observatorio de Marina, por entonces primer meridiano español, sino también que se calculasen directamente en España. Asimismo resultaba deseable una construcción autóctona de los instrumentos de reflexión necesarios para su aplicación, algo que también se podría decir de los cronómetros marinos.

Adquiridos los instrumentos, asimilados los conocimientos necesarios para su empleo, el último paso en la apropiación de las que podríamos calificar de nuevas tecnologías diseñadas para la determinación directa de la longitud geográfica en el mar era la consecución de la total independencia respecto del extranjero. Se registran cuatro intentos de la Marina en este sentido. Por un lado, la formación en el seno del Observatorio de una Oficina de Calculadores que confeccionase las efemérides españolas, algo que se consiguió, publicándose el primer Almanaque Náutico en 1791, con tablas de distancias lunares para el año 1792 y otras de utilidad general para el navegante; pese a los tiempos difíciles que se avecinaban, tal publicación no se vio en ningún momento interrumpida, prosiguiéndose en la actualidad. Dificultades más serias obstaculizaron los otros tres intentos. Uno de ellos tuvo que ver con el establecimiento de un taller de construcción de telescopios; otro, con un Obrador de Relojería, destinado al mantenimiento y construcción de cronómetros marinos; finalmente, un último intento tuvo que ver con un taller análogo para la construcción, entre otros, de instrumentos de reflexión.

En el primer caso, la Marina tuvo noticias de la existencia de un constructor de telescopios (reflectores), el presbítero Vicente Assensio, a través del anuncio que éste puso en la Gaceta de Madrid del 11 de abril de 1787. Como prueba, a Assensio se le solicitó la construcción de un telescopio con determinadas especificaciones técnicas, para lo cual se le asignaría una pensión de 3 o 4.000 reales, que Assensio debería devolver si el trabajo no se llevaba a cabo con resultados satisfactorios. El resultado no sería todo lo bueno que se esperaba, pero aún así a Assensio se le abonaron 13.325 reales y $15 \mathrm{mrs}$. por el trabajo. Assensio pretendió que se le estableciese un taller-escuela, pero los dudosos resultados redujeron la oferta de la Marina a adquirir durante diez años todos los telescopios aceptables que fabricase, abonándole en el caso de los cinco primeros el triple de su valor en Londres, y en el de los cinco siguientes el doble. No parece que finalmente esto diese resultado, y aunque a Assensio se le asignó una pensión sobre el Obispado de Canarias, aquél acabó negándose a 
trabajar en la limpieza de los espejos y reparación de los telescopios de la Marina alegando que dicha pensión no se le había llegado a pagar. ${ }^{23}$

En el caso de los cronómetros de longitud, ya Juan, en su informe de 1765, hab́a advertido de la necesidad de contar no sólo con las máquinas, sino con operarios capaces de llevar a cabo su mantenimiento:

Puede ser que ahora en los principios no condesciendan los ingleses en participarnos el secreto; pero es regular que después de las próximas experiencias no puedan evitarlo. Las medidas que será preciso tomar son de que vayan a su tiempo dos o tres relojeros españoles de los que se conocen aplicados a que aprendan con el mismo Harrison, procurando contentar a éste; pues aunque llegue el caso de que se nos vendan los cronómetros no es esto suficiente; es preciso que haya después quien nos los tenga limpios y corrientes: porque en esto consiste el beneficio, y que si llegase el caso que se rompa una rueda, haya quien la sepa hacer de nuevo. ${ }^{24}$

Con ocasión de la compra de los primeros cronómetros a F. Berthoud, éste, en 1775, se ofreció para formar un pensionado, pero su petición fue desatendida. Diez años después, todos los relojes necesitaban una limpieza, y algunos reparación. No hallándose en Cádiz quien pudiese hacerlo satisfactoriamente, hubo que enviar los relojes al taller del constructor, adonde llegaron muy maltratados por el viaje. Esto sucedía en 1788, momento en que Berthoud, a la vista de lo sucedido, renovaba la oferta de la formación de un pensionado que se ocupase en Cádiz del mantenimiento de los cronómetros. De nuevo la renovó Berthoud al año siguiente, pero esta vez añadió el importante matiz de enseñar al pensionado que se le enviase no sólo el mantenimiento de los relojes, sino el oficio de constructor, poniéndolo en condiciones de fabricarlos por sí mismo. Y esta vez la propuesta fue atendida rápidamente.

${ }^{23}$ El telescopio de Assensio se conserva en el Museo Naval de Madrid. Sobre el tema, véase Vicente VELA, "Un telescopio espafiol del siglo XVIII", Arbor, 31 (1955), 462-475. Asimismo A. LAFUENTE y M. Selles, El Observatorio..., op. cit., págs. 274-280.

${ }^{24} \mathrm{El}$ informe se recoge en su totalidad en C. FERNANDEZ DURO, "Cronometría", en Disquisiciones náuticas, vol. 4, Madrid, 1879, págs. 83-191; también en J. EsPINOSA TELlo, Memorias sobre las observaciones astronómicas hechas por los navegantes españoles en distintos puntos del globo, 2 vols., Madrid, 1809, vol. 1, págs. 176-184. 
Para pupilo de Berthoud fue escogido Cayetano Sánchez, joven alumno de la Escuela de Relojería que los hermanos Charost habían establecido en Madrid. En las condiciones estipuladas con Sánchez, a cambio de la obligación de su aprendizaje, se estipulaba pagar una pensión a su madre, los gastos de viaje y mantenimiento, y un sueldo de 300 reales mensuales el primer año, 400 el segundo y 500 el tercero. La enseñanza finalizó con informe favorable de Berthoud en 1791 (su salario fue de 20.000 libras tornesas) y la Marina dispuso redondear su formación enviándolo a Londres durante un año con Emery, quien recibiría por su enseñanza 200 libras esterlinas. Sánchez retornó a Cádiz a fines de 1792, provisto de las máquinas-herramienta y otros útiles que tanto Berthoud, por un lado, como Emery, por otro, consideraron necesarias para la construcción de cronómetros. $\mathrm{El}$ contrato que en esta ocasión firmó con la Marina incluía el establecimiento de un taller en la Isla de León y un sueldo de 1.000 reales mensuales, a cambio de lo cual debía ocuparse del mantenimiento y reparación de los cronómetros, relojes y péndulos de la Armada o de propiedad particular de sus oficiales, sólo por el coste de los materiales empleados. También debería construir los cronómetros que se le solicitasen, aunque con una gratificación de medio sueldo mientras duurase el trabajo. A cambio podría establecerse para realizar trabajos particulares, adquiriéndole la Marina los que le interesasen a los precios corrientes del mercado. Se le asignó asimismo un ayudante con un sueldo de 700 reales mensuales, plaza para la que propuso Sánchez a otro alumno de la Escuela de Madrid, Eugenio Cruzado. Junto a Sánchez se colocó a otro pensionado, Antonio Molina, quien aprendería con Emery el trabajo de las piedras duras empleadas en relojería, firmando un contrato con la Marina en términos similares a los de Sánchez.

Esta vez los resultados fueron mejores, y aunque no se construyeron cronómetros nuevos, los ya existentes se repararon a completa satisfacción. Pero este taller tuvo un pronto y desgraciado final. Molina falleció en París a principios de 1798, mientras adquiría máquinas para el taller. Su sustituto fue otro pensionado parisino, Carlos la Rue, que comenzó su trabajo de forma prometedora, pero que falleció asimismo en 1800, junto con Sánchez y Cruzado, a causa de la epidemia de fiebre amarilla que asoló la región. El Obrador de Relojería quedaba vacío, y todas las esperanzas truncadas. Aunque esto no detuvo a la Marina, que renovó el intento con el envío de nuevos pensionados. ${ }^{25}$

${ }^{25}$ Esta vez a aprender con Louis Berthoud, sobrino del anterior. Los pensionados fueron Agustín Albino y Blas Muñoz, y las condiciones fueron similares a las pactadas en el anterior 
El tercer intento, por último, tuvo que ver con la construcción de instrumentos de reflexión. El protagonista fue aquí José María Baleato, un prometedor operario del obrador de instrumentos del Arsenal de Ferrol, quien partió en 1785 para Londres con el fin de instruirse durante dos años. Su retorno coincidió con una gestión importante, la adquisición de una máquina de dividir limbos graduados. Como es sabido, el primero en fabricar satisfactoriamente una máquina de este tipo fue J. Ramsden en 1768 , y ésta era la máquina que deseaba conseguir la Marina a través de J. H.Magellan. Pero, enfermo éste, se recurrió a otro agente, con el resultado de que se adquirió otra máquina de dividir, que salió bastante barata - $\mathbf{4 0}$ guineas cuando la de Ramsden se había presupuestado en 600- y cuyo constructor es desconocido. Por su parte, Baleato, de retorno en el Arsenal, había obtenido la dirección de un taller-escuela en el Arsenal, y se había ofrecido a construir por sí mismo la máquina de Ramsden. Ninguna de las dos iniciativas tuvo los resultados apetecidos. Cuando llegó el divisor de Londres, Baleato informó que no era apropiado para la división del tipo de limbos que se usaban ${ }^{26}$. Pero su máquina tampoco funcionó satisfactoriamente, presentando vibraciones que condujeron a divisiones defectuosas. Baleato fabricó varios instrumentos, entre ellos un círculo de reflexión según el modelo de Borda, que al ser examinado mostró graves defectos, además de en la división, en el paralelismo del espejo central. Una nueva remesa de instrumentos fabricados por Baleato - un quintante y un octante de metal, así como un sextante y otro octante de madera - mostraron de nuevo, salvo el quintante, una división defectuosa, y todos elllos mala calidad en los espejos que, como en el caso del círculo de Borda, se habían adquirido en Londres. No obstante, el resultado se juzgó prometedor, y se mantuvo la esperanza de que los resultados fuesen mejorando con el tiempo. Por entonces Baleato - al igual que Assensio- propuso la formación de un taller de instrumentos que surtiese a toda la Marina, pero la solicitud de este establecimiento monopolista fue desestimada al juzgarse más con-

envío, así como las pactadas a su regreso en 1806. Las rencillas entre Albino y Muñoz y las dificultades de la invasión francesa dieron al traste con el Obrador, que desapareció del Observatorio en 1818 al aceptarse la solicitud de traslado de Munoz a Madrid. Las vicisitudes del Obrador se estudian en A. LAFUENTE y M. SELLES, El Observatorio..., op. cit., págs. 321-357.

${ }^{26}$ Informaba que con ella no se podían dividir limbos de más de 11,5 pulg. de radio y 2 líneas de espesor, mientras que los que se usaban habitualmente tenían 16 pulg. y 6 líneas, respectivamente. Éste y otros detalles de la historia del Obrador de Baleato se recogen en M. SELLÉS, «El problema de la construcción de instrumentos náuticos en la España del setecientos», en $\mathrm{M}$. VAlERA y C. LOPEZ FERnÁndez (eds.), Actas del V Congreso de la Sociedad Espafiola de Historia de las Ciencias y de las Técnicas, 3 vols., Murcia: SEHCT, 1991, vol. 3, págs. 1913-1927. 
veniente que Baleato formase a un conjunto de constructores que luego, estableciéndose independientemente, instruyesen a otros nuevos y compitiesen entre sí. Así, el contrato que la Marina estableció con Baleato, similar a los dos casos anteriores ya vistos, preveía sueldos y gratificaciones. La ausencia de documentación posterior hace pensar que las cosas no fueron por buen camino, aunque consta la formación de algún discípulo, como Lorenzo Labora, que pasaría a ser instrumentario del Observatorio de la Isla de León y que fallecería también en 1800 a causa de la epidemia de fiebre amarilla.

No terminaron aquí las tentativas de la Armada. En 1791 se logró que Stancliffe aceptase la formación de un pensionado español, Miguel Borges, quien firmó un contrato en todo silimar a los estipulados en el caso del Obrador de Relojería y del Obrador de Baleato. Borges regresó en 1800 y fue enviado al Observatorio con el encargo del cuidado de sus instrumentos y de que construyese un instrumento de reflexión para demostrar su capacidad. El sextante que fabricó mostraba su habilidad en el trabajo, pero serias deficiencias en la división -a falta de máquina de dividir se había usado como patrón otro sextante-, mala calidad en los espejos —adquiridos en Cádiz_y además carecía de anteojo por la falta de máquinas apropiadas para fabricarlo. En 1805 Borges se trasladaba a Inglaterra sin pedir licencia para ello, y llegaba a su fin esta nueva tentativa.

En realidad, el período en el que se llevaron a cabo los proyectos más destacados sólo se extendió entre 1783 y 1795 . A partir de este último año la Marina de la Ilustración inició su declive, dentro de un clima de mala administración y penurias económicas. En 1793-95 se dio la guerra con la Convención francesa y, a partir de 1796, las alianzas cambiadas, contra Inglaterra, rematándose estas contiendas bélicas con la Guerra de la Independencia. Realmente se trataba de un plazo muy breve para la realización de unos programas tan importantes, de modo que los resultados alcanzados, aunque parciales, fueron sorprendentes.

Para finalizar, quiero recoger aquí la observación con que iniciaba estas páginas, relativa a que la historia de un instrumento, o un determinado conjunto de los mismos, puede y debe incluir también su apropiación y uso por un colectivo. Apropiación tanto intelectual como física, dos aspectos que conviene distinguir pues, como se ha visto, ambos muestran dependencias muy distintas. La apropiación intelectual depende de una adecuada estructura docente y con contenidos apropiados. Caso de no existir tal estructura, deberá crearse al intento, para lo cual se precisan fondos y el recurso externo a un pro- 
fesorado lo suficientemente competente; los contenidos comportan algo más sencillo, una reforma en los planes de estudios. Dentro de la apropiación física podrían considerarse dos fases: una, evidentemente, es la adquisición de los instrumentos pertinentes en los centros de producción del extranjero; la otra, el establecimiento de centros de producción propios. Aquí intervienen factores más delicados pues, aparte del adiestramiento técnico de los artífices, la consecución de esto se apoya en toda una infraestructura tecnológica que implica desde las apropiadas materias primas a las máquinas-herramienta. En ambos casos se supone la existencia de una demanda, particularmente en el segundo de un mercado, que sometido a las leyes de la oferta y la demanda debe ser competitivo. Como se ha visto, en España no se dieron las condiciones para esto último.

En cuanto al uso, éste resulta decisivo para el desarrollo del conocimiento cientifico o para la aplicación de una tecnología, como es el caso de la navegación. Ateniéndonos a este último aspecto cabe decir que, simplemente, o bien existe una demanda consolidada, o se debe dar una adecuada planificación de objetivos -hoy lo llamamos política científica y tecnológica-, caso que resulta patente a la vista de la escasa utilización que hizo la Marina de la dotación instrumental del Observatorio en sus primeras décadas de existencia. Respecto de este punto, resulta curioso observar - y quizás deba ser objeto de alguna reflexión- que tanto el Observatorio de Greenwich, cuyo propósito fundacional estaba relacionado con el problema de la determinación de la longitud, como el de Cádiz, fundado en el seno de la Marina, no alcanzasen a lograr las tablas del movimiento lunar necesarias para la aplicación del método de las distancias lunares, cuando sí se hizo desde un Observatorio desvinculado de connotaciones náuticas, como lo era el de Gotinga. Como se ha visto, en el caso del Observatorio de Cádiz este propósito ni tan siquiera llegó a plantearse. Permítaseme apuntar entre las posibles causas el hecho de que, aparte de que la Armada espaniola tuviese sus propias urgencias, las observaciones nunca estuvieron a cargo de personal civil que, desprovisto de responsabilidades militares, pudiese dedicarse con continuidad a la tarea — supuesto que ésta se le hubiese señalado-, así como el de que se fundó con objetivos, aunque vagos, en los que la docencia tenía un papel destacado, papel que, como se ha visto aquí, se desempeñó cumplidamente.

Parece, pues, que existe una serie inevitable de pasos en la apropiación tecnológica, pasos que dio a su tiempo la Marina española hasta llegar, en la última parte del período considerado, a realizar aportaciones originales, aun cuando 
éstas fuesen de índole teórica ${ }^{27}$. Pero la parte técnica no se consiguió, al no bastar con la voluntad política, manifestada en la fundación de talleres-escuela: en España faltaba toda la infraestructura necesaria, algo que podría ponerse en consonancia con la práctica ausencia en la época de una clase burguesa comercialmente emprendedora cuyas actividades y demanda hubiesen podido crearla.

${ }^{27}$ El ejemplo destacado lo constituyen las conocidas tablas elaboradas por José de Mendoza y Ríos para facilitar la aplicación del método de las distancias lunares, que fueron de uso bastante general en el siglo XIX. Sobre ésta y otras aportaciones, véase M. A. SELLÉS, Navegación astronómica... op. cit., págs. 261 ss. 